\title{
Apparent oliguria following urological surgery
}

\author{
${ }^{1} \mathrm{JS}$ Murray, ${ }^{1} \mathrm{ES}$ Wijewickrama, ${ }^{2} \mathrm{P}$ Haslam, ${ }^{3} \mathrm{NS}$ Kanagasundaram \\ ${ }^{1}$ Specialist Registrar, Nephrology, Renal Services, Newcastle upon Tyne Hospitals NHS Foundation Trust; ${ }^{2}$ Consultant Radiologist, \\ Department of Radiology, Newcastle upon Tyne Hospitals NHS Foundation Trust; ${ }^{3}$ Consultant Nephrologist, Newcastle upon Tyne Hospitals \\ NHS Foundation Trust, UK and Honorary Clinical Senior Lecturer, Institute of Cellular Medicine, Newcastle University, UK
}

\begin{abstract}
Oliguria is a common feature of acute kidney injury $(\mathrm{AKI})$, but should be interpreted in the context of other biochemical markers when diagnosing and monitoring AKI or considering the need for renal support. We report an unusual case of apparent severe oliguria arising as a result of complex urological pathology and discuss how an understanding of creatinine kinetics raised suspicions of an alternative diagnosis. We discuss the problems caused by an over-reliance on urine output or serum creatinine alone when diagnosing and staging $\mathrm{AKI}$ and highlight the need for a more holistic approach.
\end{abstract}

KEYWORDS Normal anion gap metabolic acidosis, renal replacement therapy, renal cell carcinoma, transitional cell carcinoma, urinary fistula

DECLARATION OF INTERESTS No conflicts of interest declared.

\author{
Correspondence to \\ NS Kanagasundaram \\ Renal Services, Freeman Hospital \\ High Heaton, \\ Newcastle Upon Tyne \\ NE3 4YN, UK \\ tel. +44 (0)191 2137149 \\ e-mail \\ suren.kanagasundaram@nuth.nhs.uk
}

\section{INTRODUCTION}

Oliguria is a common feature of acute kidney injury $(\mathrm{AKI})$, but should be interpreted in the context of other biochemical markers when diagnosing and monitoring $\mathrm{AKI}$, or considering the need for renal support. This is particularly true in patients with a history of renal tract or pelvic disease or surgery.

\section{CASE REPORT}

A 57-year-old man required emergency admission with a six-week history of profuse diarrhoea. He had a complex urological history: eight months prior to admission he had required drainage of a left renal bed collection, which had developed following a partial left nephrectomy for renal cell carcinoma; three months prior to admission, he had also undergone right total nephroureterectomy for transitional cell carcinoma; his left ureteric stent was replaced two months before admission after a renocutaneous fistula between the left middle calyx and skin was identified as the cause of the discharge of purulent urine from a previous surgical drain site; five weeks prior to the current admission, his serum creatinine had remained stable at 260 micromoles per litre $(\mu \mathrm{mol} / \mathrm{L})$.

On admission the patient looked unwell, was confused and unsteady. His renocutaneous fistula was discharging purulent urine. He had AKI (serum creatinine now 337 $\mu \mathrm{mol} / \mathrm{L})$ and severe metabolic acidosis $(\mathrm{pH} 7.13)$, which was shown to be of a normal anion gap on day three of his admission (venous bicarbonate 18 millimoles per litre [mmol/L], Cl- $120 \mathrm{mmol} / \mathrm{L}, \mathrm{Na}+147 \mathrm{mmol} / \mathrm{L}, \mathrm{K}+3.3$ $\mathrm{mmol} / \mathrm{L}$ ). His initial urine output (measured via a urethral catheter) ranged between 150-200 millilitres per hour
(mL/hr). An ultrasound scan showed no hydronephrosis. His AKI was provisionally ascribed to ischaemic acute tubular necrosis (ATN) due to volume depletion from diarrhoea. Continuous veno-venous haemofiltration was instituted on critical care, primarily for correction of his acidaemia. His serum creatinine had fallen to $275 \mu \mathrm{mol} / \mathrm{L}$ when renal support was suspended, six hours prior to step down to the renal unit on day two of his admission.

The following day, it was noted that his urine output had fallen to $246 \mathrm{~mL}$ in the preceding 24 hours. A second renal tract ultrasound again showed no obstruction. Despite being oliguric, the patient's serum creatinine fluctuated between 272 and $304 \mu \mathrm{mol} / \mathrm{L}$ between days three and eight of his admission, without evidence of a progressive rise - a finding seemingly incompatible with the clinical diagnosis of ongoing, oliguric, ischaemic ATN (Figure I). Although noted as being 'watery', changes in the nature of his diarrhoea in the initial stages of his admission were not documented so a relationship between this and his urine output could not be ascertained; however, on day eight he was noted to have passed 'firm stool mixed with urine'. Mindful of the patient's past urological history, reinstitution of renal support was deferred and further investigations arranged. A fistulogram was undertaken on day ten of his admission which showed the presence of a reno-colonic fistula, explaining his 'watery diarrhoea', his apparent oliguria and his stable renal excretory capacity (Figure 2).

The patient subsequently underwent left hemi-colectomy and disconnection of his reno-colonic fistula with later plugging of his reno-cutaneous tract. Unfortunately, his chronic kidney disease ultimately progressed to end-stage renal disease and dependence on maintenance dialysis. 


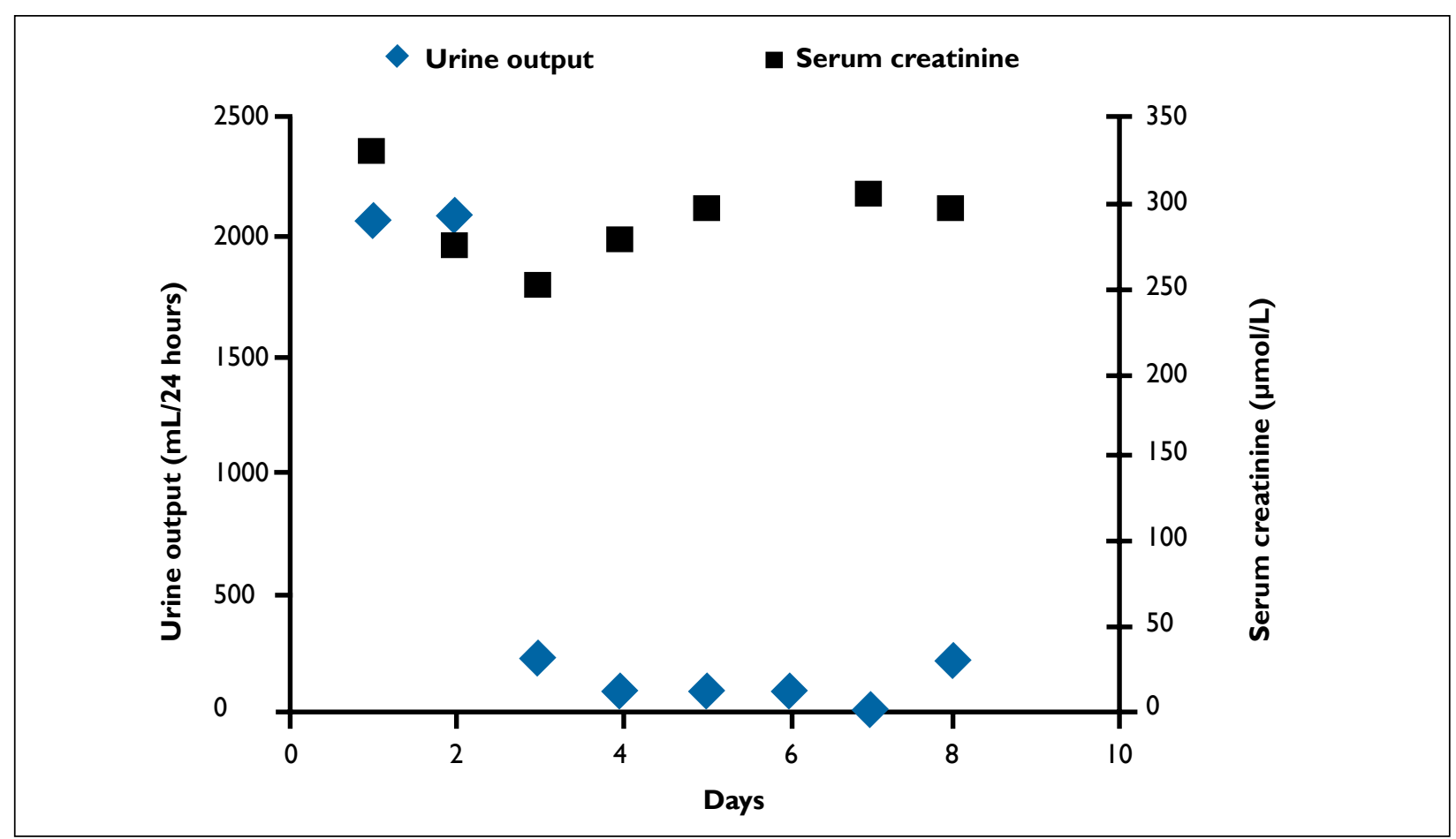

FIGURE I Serum creatinine and urine output changes since admission. The initial fall in serum creatinine reflected treatment with continuous haemofiltration prior to step down to the renal ward on admission day two. Despite the presence of oliguria between days three and eight, serum creatinine remained relatively stable.

\section{DISCUSSION}

Ureterocolonic fistula formation is a rare cause of apparent oliguria and watery diarrhoea.' In our case, an understanding of expected biochemical changes in oliguric ATN highlighted the incompatibility between this diagnosis and evidence of a stable serum creatinine. Current consensus criteria for AKI allow both diagnosis and stratification of severity through changes in either serum creatinine or urine output alone. ${ }^{2}$ A variety of factors can influence criteria based on serum creatinine (for instance muscle mass, the use of drugs that interfere with the tubular secretion of creatinine and supplemental creatine). ${ }^{3}$ It is also recognised, however, that oliguria can occur for reasons other than AKI. For example, the hormonal response to trauma or major surgery can result in antidiuresis and oliguria. ${ }^{4}$ An incompatible clinical history and the persistence of oliguria beyond 24 hours ${ }^{4}$ would go against this aetiology in the present case, however. It has also been suggested that the strict definition of $A K I$ requires the correction of pre-renal azotaemia $^{5}$ - the reduction in renal excretory capacity arising from renal under-perfusion. ${ }^{6}$ Pre-renal azotaemia is a predictable by-product of a normal, physiological response to reduced effective circulating volume and is readily reversed through volume replacement or vasopressor therapy as no renal cell injury (i.e. AKI) has yet occurred. In our case, an understanding that renal excretory capacity is maintained by an obligatory urine output of around $500 \mathrm{~mL} / \mathrm{day}^{7}$ would have highlighted the incompatibility with both 'pre-renal' azotaemia and established $\mathrm{AKI}$; in a persisting and severe oliguric state, one would expect progressive accumulation of excretory products such as creatinine. Generated from muscle metabolism of creatine, ${ }^{3}$ the magnitude of the serum creatinine rise would not just reflect the profundity of

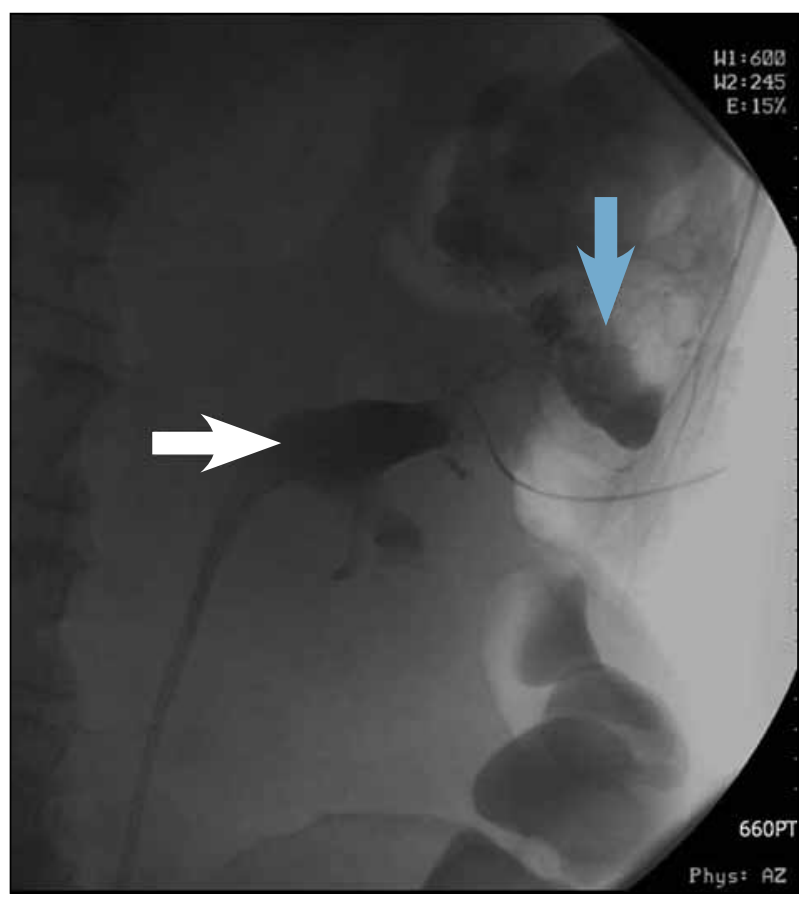

FIGURE 2 Fistulogram showing contrast in the left renal pelvis (white arrow) and the descending colon (blue arrow) demonstrating the reno-colonic fistula. 
renal dysfunction but also the creatinine generation rate, the underlying muscle mass and the volume of distribution of creatinine. Mathematical modelling ${ }^{8}$ has suggested that in severe $\mathrm{AKI}$, defined as a $90 \%$ reduction in creatinine clearance from baseline, one would expect serum creatinine to rise by around 160 to $175 \mu \mathrm{mol} / \mathrm{L} /$ day. A more modest $50 \%$ reduction in creatinine clearance would yield a serum creatinine rise of around $50 \mu \mathrm{mol} / \mathrm{L} /$ day.

The presence of a normal anion gap metabolic acidosis could have been compatible with the patient's diarrhoea, regardless of the presence or absence of his renocolonic fistula. However, the return of renal function to the pre-morbid baseline and the lack of any subsequent significant change in serum creatinine, despite persisting, severe oliguria, suggested that this was not simply prerenal azotaemia or ischaemic AKI.

\section{REFERENCES}

I Punga-Maole A, Mbongo BA, Molua AA et al. [Postoperative ureterocolonic fistula: a case report]. Prog Urol 2005; 15:93-5. French.

2 Lewington A, Kanagasundaram S. Renal Association clinical practice guidelines on acute kidney injury. Nephron Clin Pract 201 I; I I8:c349-90. http://dx.doi.org//0.I I59/000328075

3 Refaie R, Moochhala SH, Kanagasundaram NS. How we estimate GFR - a pitfall of using a serum creatinine-based formula. Clin Nephrol 2007; 68:235-7.

4 Powell-Tuck J, Gosling P, Lobo DN et al. British consensus guidelines on intravenous fluid therapy for adult surgical patients (GIFTASUP) [Internet]. 20II [cited 2012 July I3]. Available from: http://www.bapen.org.uk/pdfs/bapen_pubs/giftasup.pdf

5 Mehta RL, Kellum JA, Shah SV et al. Acute Kidney Injury Network: report of an initiative to improve outcomes in acute kidney injury. Crit Care 2007; I I:R3 I. http://dx.doi.org/I0.1 I86/cc57I3

\section{CONCLUSION}

Although our case represents a rare cause of oliguria it highlights the importance of an holistic approach to the diagnosis of $\mathrm{AKI}$ - especially given the treatable underlying diagnosis and the risks of renal support.' Consensus definitions, from the RIFLE criteria, ${ }^{10}$ through Acute Kidney Injury Network $(A K I N)^{5}$ to the most current diagnostic system ${ }^{2}$ are powerful tools for understanding the epidemiology of AKI. Despite potential pitfalls, careful consideration of clinical context, effective circulating volume and the interplay between creatinine kinetics and urine output should provide the clearest insight into individual underlying pathophysiology.

6 Griffiths L, Kanagasundaram NS.Assessment and initial management of acute kidney injury. Medicine 20II; 39:390-7. http://dx.doi. org/l0.1016/j.mpmed.201 I.04.010

7 Guyton AC, Hall JE. Textbook of medical physiology. 9th ed. Philadelphia:WB Saunders Company; 1996. p. 298.

8 Waikar SS, Bonventre JV. Creatinine kinetics and the definition of acute kidney injury. J Am Soc Nephrol 2009; 20:672-9. http://dx.doi. org/I0.168I/ASN.2008070669

9 Kanagasundaram NS. Renal replacement therapy in acute kidney injury: an overview. Br J Hosp Med 2007; 68:292-7.

I0 Bellomo R, Ronco C, Kellum JA et al. Acute renal failure definition, outcome measures, animal models, fluid therapy and information technology needs: the Second International Consensus Conference of the Acute Dialysis Quality Initiative (ADQI) Group. Crit Care 2004; 8:R204-12. http://dx.doi.org/I0.II86/cc2872 